\title{
Injective comodules and Landweber exact homology theories
}

\author{
by \\ Mark Hovey (Middletown, CT)
}

\begin{abstract}
We classify the indecomposable injective $E(n)_{*} E(n)$-comodules, where $E(n)$ is the Johnson-Wilson homology theory. They are suspensions of the $J_{n, r}=$ $E(n)_{*}\left(M_{r} E(r)\right)$, where $0 \leq r \leq n$, with the endomorphism ring of $J_{n, r}$ being $\widehat{E(r)}^{*} \widehat{E(r)}$, where $\widehat{E(r)}$ denotes the completion of $E(r)$.
\end{abstract}

Introduction. Perhaps the most important homology theories in algebraic topology are complex bordism $M U$ and the many theories derived from it. In particular, if $p$ is a prime, and we localize at $p$ as we do throughout this paper, then $M U$ splits as a coproduct of suspended copies of a spectrum known as $B P$, with $B P_{*}(*) \cong \mathbb{Z}_{(p)}\left[v_{1}, v_{2}, \ldots\right]$, where $v_{i}$ has degree $2\left(p^{i}-1\right)$. Derived from $B P$ are the many Landweber exact homology theories [Lan76], such as the Johnson-Wilson theory $E(n)$, with $E(n)_{*}(*) \cong \mathbb{Z}_{(p)}\left[v_{1}, \ldots, v_{n}, v_{n}^{-1}\right]$ and Morava $E$-theory $E_{n}$ (see [DH95]).

As is well-known [Rav86, Theorem 2.2.8], for all of these homology theories $h$, the homology groups $h_{*} X$ form a graded comodule over the graded Hopf algebroid $\left(h_{*}, h_{*} h\right)$. It therefore behooves us to learn as much as possible about the category of $h_{*} h$-comodules. In particular, products are not exact in the category of $h_{*} h$-comodules, and their derived functors form the $E_{2}$-term of a spectral sequence [Hov07] converging to the $h_{*}$-homology of a product of spectra. This spectral sequence offers one possible approach to the chromatic splitting conjecture of Hopkins [Hov95].

The author has thus been engaged in attempting to understand as much of the structure of the category of $h_{*} h$-comodules as possible, following in the footsteps of Peter Landweber, whose papers [Lan76], [Lan73], and the less well-known but excellent [Lan79] are the basis for our understanding of $B P_{*} B P$-comodules. In particular, the author and Strickland, in [HS05a]

2000 Mathematics Subject Classification: 55N22, 55S25, 13C11, 16W30.

Key words and phrases: injective comodule, Landweber exact homology theory. 
and [HS05b], have shown that analogues of Landweber's theorems hold in the category of $E_{*} E$-comodules, where $E$ denotes, as it will throughout the paper, a Landweber exact homology theory of height $n$ such as $E(n)$ or $E_{n}$. Recall that this means that there is a ring homomorphism $B P_{*} \rightarrow E_{*}$ so that the sequence $\left(p, v_{1}, \ldots, v_{n}\right)$ is regular in $E_{*}$ and $v_{n}$ is a unit in $E_{*} /\left(p, v_{1}, \ldots, v_{n-1}\right)$.

The present paper is devoted to the study of injective $B P_{*} B P$-comodules and $E_{*} E$-comodules. Injective comodules have not been studied before, except briefly in [HS05b], because one can almost always use relatively injective comodules to compute the derived functors of interest in topology. This includes the Ext groups $\operatorname{Ext}_{E_{*} E}\left(E_{*}, E_{*} X\right)$ and even the derived functors of product.

However, we will show in this paper that the injective comodules have a very rigid structure, in analogy to the Matlis theory of injective modules over a Noetherian commutative ring (described in [Lam99, Section 3I]). There are only $n+1$ different isomorphism classes of indecomposable injective $E_{*} E$ comodules, where $E$ is, as always, a Landweber exact homology theory of height $n$. They are the injective hulls $J_{r}$ of $E_{*} / I_{r}$, where $0 \leq r \leq n$ and $I_{r}=\left(p, v_{1}, \ldots, v_{r-1}\right)$. Furthermore, if $E_{*}$ is evenly graded, $J_{r} \cong E_{*}\left(M_{r} E(r)\right)$ up to suspension, where $M_{r}$ is the fiber of the map of Bousfield localizations $L_{r} \rightarrow L_{r-1}$. Also, the endomorphism ring of $J_{r}$ as an $E_{*} E$-comodule is $\widehat{E(r)}^{*} \widehat{E(r)}$, where $\widehat{E(r)}=L_{K(r)} E(r)$ is the completion of $E(r)$ at $I_{r}$.

Unfortunately, a good algebraic description of $\widehat{E(r)}^{*} \widehat{E(r)}$ is not known. We know that $E_{r}^{*} E_{r}$ is the twisted completed group ring $E_{r *}\left[\left[S_{r}\right]\right]$ on the large Morava stabilizer group (this is an old result of Hopkins and Ravenel; see [Bak89] or [Hov04b] for proofs of it). It therefore seems likely that $\widehat{E(r)}^{*} \widehat{E(r)}$ is very closely related to the stabilizer group $S_{r}$. If we accept this, then we are seeing all of the stabilizer groups $S_{r}$ for $0 \leq r \leq n$ in the category of $E_{*} E$-comodules. Since the chromatic splitting conjecture is in some sense about how the apparently unrelated stabilizer groups actually are related to each other, seeing all of the stabilizer groups together like this is a good sign and might be useful. We can also find a decomposable injective comodule whose endomorphism ring is $E_{r}^{*} E_{r}$.

As a corollary of our work, we rediscover the folklore isomorphisms

$$
\begin{aligned}
\widehat{E(n)}^{*}(X) & \cong \operatorname{Hom}_{E(n)_{*}}\left(E(n)_{*}\left(M_{n} X\right), \Sigma^{-n} E(n)_{*} / I_{n}^{\infty}\right), \\
E_{n}^{*} X & \cong \operatorname{Hom}_{E_{n *}}\left(E_{n *}\left(M_{n} X\right), \Sigma^{-n} E_{n *} / I_{n}^{\infty}\right) .
\end{aligned}
$$

As far as the author knows, these isomorphisms have not been written down before, though they were certainly known to Hopkins, Greenlees, Sadofsky, and others. 
Throughout this paper, $p$ will be a fixed prime integer, all spectra will be localized at $p$, and $n>0$ will be a fixed positive integer. The symbol $E$ will denote a Noetherian Landweber exact homology theory of height $n$, like $E(n)$ or $E_{n}$. The symbol $(A, \Gamma)$ will denote a flat Hopf algebroid.

The author thanks Andy Baker for sharing his insights into $\widehat{E(r)} * \widehat{E(r)}$.

1. $B P_{*} B P$-comodules and $E_{*} E$-comodules. The purpose of this section is to remind the reader of some of the results on the structure of $B P_{*} B P-$ comodules, $E_{*} E$-comodules, and the relation between them.

Recall that the Hopf algebroid $(A, \Gamma)$ is called an Adams Hopf algebroid when $\Gamma$ is the colimit of a filtered system of comodules $\Gamma_{i}$, where each $\Gamma_{i}$ is finitely generated and projective over $A$. As explained in [Hov04a, Section 1.4] (though originally due to Hopkins), both $\left(B P_{*}, B P_{*} B P\right)$ and $\left(E_{*}, E_{*} E\right)$ are Adams Hopf algebroids. When $(A, \Gamma)$ is an Adams Hopf algebroid, the category of $\Gamma$-comodules is a Grothendieck category [Ste75, Chapter V]. This means that it is an abelian category in which filtered colimits are exact, and that there is a family of generators $\left\{P_{i}\right\}$, in the sense that $\Gamma-\operatorname{comod}\left(\bigoplus P_{i},-\right)$ is a faithful functor. The most natural collection of generators consists of the dualizable comodules; these are the comodules that are finitely generated and projective over $A$. These are studied and proved to be generators in Sections 1.3 and 1.4 of [Hov04a]. Note that $B P_{*}$ and its suspensions do not generate the category of $B P_{*} B P$-comodules, and we know of no naturally defined proper subcollection of the dualizable comodules which does so.

As is well-known, the category of $\Gamma$-comodules is a closed symmetric monoidal category [Hov04a, Section 1.3]. The tensor product is denoted $M \wedge N$; it is isomorphic as an $A$-module to the tensor product $M \otimes_{A} N$ of left $A$-modules (a different notation is used because the usual tensor product symbol is reserved for the tensor product of $A$-bimodules, which occurs frequently in the theory). The internal Hom object is denoted $F(M, N)$; there is a natural map $F(M, N) \rightarrow \operatorname{Hom}_{A}(M, N)$ of $A$-modules that is an isomorphism when $M$ is finitely presented over $A$.

There are enough injectives in any Grothendieck category. One can see this directly for $\Gamma$-comodules by noting that $\Gamma \otimes_{A} J$ is an injective $\Gamma$ comodule whenever $J$ is an injective $A$-module. Note that this is a bimodule tensor product, and all the $\Gamma$-coaction is concentrated on the $\Gamma$ tensor factor; however, it is well-known that $\Gamma \otimes_{A} J$ is isomorphic as a comodule to $\Gamma \wedge J$ (see [Hov04a, Lemma 1.1.5]). Moreover, if $A$ is Noetherian, as it is for $(A, \Gamma)=\left(E_{*}, E_{*} E\right)$, the category of $\Gamma$-comodules is locally Noetherian as well. This means that subcomodules of the generators satisfy the ascending chain condition; this is obvious because dualizable comodules are 
finitely generated over $A$. In any locally Noetherian Grothendieck category, direct sums of injectives are injective and every injective is a direct sum of indecomposable injectives in an essentially unique way (see [Ste75, Sections V.4, V.5]).

There is a functor $\Phi_{*}$ from $B P_{*} B P$-comodules to $E_{*} E$-comodules defined by $\Phi_{*} M=E_{*} \otimes_{B P_{*}} M$. This functor is much studied in [HS05a] and [HS05b]. The functor $\Phi_{*}$ is exact and has a right adjoint $\Phi^{*}$. The composite $\Phi_{*} \Phi^{*}$ is naturally equivalent to the identity, and the composite $\Phi^{*} \Phi_{*}$ is the localization functor $L_{n}$ with respect to the hereditary torsion theory of $v_{n}$-torsion comodules. In particular, $\Phi^{*}$ defines an equivalence of categories between $E_{*} E$-comodules and $L_{n}$-local $B P_{*} B P$-comodules. This result is valid even if $E_{*}$ is not Noetherian, so the results in this paper about injective $E_{*} E$ comodules also apply to the case when $E_{*}$ is not Noetherian. It is proved in [HS05b, Section 2] that $\Phi_{*}$ and $\Phi^{*}$ preserve injectives, filtered colimits, and arbitrary direct sums. In fact, if $T_{n} M$ denotes the subcomodule of $v_{n^{-}}$ torsion elements in $M$, then $T_{n}$ also preserves injectives, and $L_{n} J=J / T_{n} J$ for injective $B P_{*} B P$-comodules $J$.

2. Indecomposable injectives. We have remarked above that every injective $E_{*} E$-comodule is a direct sum of indecomposable injectives. Matlis has a well-known theory of indecomposable injectives over Noetherian rings (see, for example, [Lam99, Section 3I]), and we will mimic his theory for indecomposable injective $E_{*} E$-comodules and $B P_{*} B P$-comodules. The object of this section is to classify all the indecomposable injective $E_{*} E$-comodules.

We first point out that indecomposable injectives are relevant even for $B P_{*} B P$-comodules.

Proposition 2.1. Suppose $J$ is an injective $B P_{*} B P$-comodule for which there exists an $n$ such that $J$ has no $v_{n}$-torsion. Then $J$ is a direct sum of indecomposable injectives in an essentially unique way.

Before proving this proposition, we point out the following lemma.

Lemma 2.2. If $N$ is an $E_{*} E$-comodule, then $N$ is indecomposable if and only if $\Phi^{*} N$ is indecomposable.

Proof. In general, an object $M$ in an abelian category $\mathcal{C}$ is indecomposable if and only if the $\operatorname{ring} \mathcal{C}(M, M)$ has no nontrivial idempotents. Since $\Phi^{*}$ is fully faithful, the result follows.

Proof of Proposition 2.1. Since $J$ has no $v_{n}$-torsion and $J$ is injective, $J$ is $L_{n}$-local. Hence $J=\Phi^{*} \Phi_{*} J$. Now $\Phi_{*} J$ is an injective object in the category of $E_{*} E$-comodules, and hence $\Phi_{*} J \cong \bigoplus J_{\alpha}$, where each $J_{\alpha}$ is an indecomposable injective $E_{*} E$-comodule. Since $\Phi^{*}$ preserves direct sums, 
we get

$$
J \cong \bigoplus \Phi^{*} J_{\alpha}
$$

Since $\Phi^{*}$ preserves injectives, and also indecomposables by Lemma 2.2, we see that $J$ is a direct sum of indecomposable injectives. The uniqueness of this direct sum decomposition follows from the Krull-Remak-SchmidtAzumaya theorem as in Section V.5 of [Ste75], using the fact that the endomorphism ring of an indecomposable injective is always local [Ste75, Proposition V.5.1].

The next thing to do is to enumerate all the indecomposable injectives. Recall that the indecomposable injective modules over a commutative Noetherian ring $R$ are the injective hulls of the $R / \mathfrak{p}$, where $\mathfrak{p}$ is a prime ideal in $R$. The ideals $I_{r}=\left(p, v_{1}, \ldots, v_{r-1}\right)$ are the prime invariant ideals in $E(n)_{*}$ for $0 \leq r \leq n$ and in $B P_{*}$ for $0 \leq r \leq \infty$ (see [HS05a, Theorem 5.6] for the $E(n)_{*}$ case). For an arbitrary Landweber exact theory of height $n$, it is possible that the $I_{r}$ are not actually prime, but they remain the "categorically prime" invariant ideals in $E_{*}$, as explained in [HS05a, Theorem 5.6]. Hence we let $J_{n, r}$ for $0 \leq r \leq n$ denote the injective hull of $E_{*} / I_{r}$ in the category of $E_{*} E$-comodules, and let $J_{r}$ for $0 \leq r \leq \infty$ denote the injective hull of $B P_{*} / I_{r}$ in the category of $B P_{*} B P$-comodules.

LEMma 2.3. The injective comodules $J_{n, r}$ for $0 \leq r \leq n$ and $J_{r}$ for $0 \leq r \leq \infty$ are indecomposable.

Proof. According to [Ste75, Proposition V.2.8], it suffices to show that $E_{*} / I_{r}$ and $B P_{*} / I_{r}$ are coirreducible, which means that any two nontrivial subcomodules $M, N$ have nontrivial intersection. This is obvious for $B P_{*} / I_{\infty}=\mathbb{F}_{p}$. For the other cases, we use the fact that every nontrivial $B P_{*} B P$-comodule or $E_{*} E$-comodule has a nonzero primitive [HS05a, Theorem 5.1]. For example, assume $0<r<n$. Then the primitives in $E_{*} / I_{r}$ are isomorphic to $\mathbb{F}_{p}\left[v_{r}\right]$ by $[\mathrm{HS} 05 \mathrm{a}$, Theorem 5.2]. Hence $M$ contains all sufficiently high powers of $v_{r}$, as does $N$, and so $M \cap N \neq 0$. The other cases are similar, using the computations of the primitives in $B P_{*} / I_{r}$ [Rav86, Theorem 4.3.2] and in $E_{*} / I_{r}$ [HS05a, Theorem 5.2].

We also note that $J_{r}$ has no $v_{r}$-torsion in view of [HS05b, Theorem 2.7]. (We will see below that $J_{n, r}$ also has no $v_{r}$-torsion.)

ThEOREM 2.4. An $E_{*} E$-comodule $J$ is an indecomposable injective comodule if and only if $J \cong \Sigma^{t} J_{n, r}$ for some $0 \leq r \leq n$ and some $t$. Similarly, a $B P_{*} B P$-comodule with no $v_{n}$-torsion is an indecomposable injective comodule if and only if $J \cong \Sigma^{t} J_{r}$ for some $0 \leq r \leq n$ and some $t$.

There are probably many different indecomposable injective $B P_{*} B P$ comodules that are $v_{n}$-torsion for all $n$. 
Proof. Suppose $J$ is an indecomposable injective $E_{*} E$-comodule. Then $J$ has a nonzero subcomodule that is finitely generated over $E_{*}$ (see [Hov04a, Proposition 1.4.1]). By the filtration theorem [HS05a, Theorem 5.7], any nontrivial finitely generated $E_{*} E$-comodule contains an isomorphic copy of $\Sigma^{t} E_{*} / I_{r}$ for some $t$ and some $r$ with $0 \leq r \leq n$. Since $J$ is injective, we see that $\Sigma^{t} J_{r} \subseteq J$; since $J$ is also indecomposable, $\Sigma^{t} J_{r} \cong J$.

Similarly, suppose $J$ is an indecomposable injective $B P_{*} B P$-comodule with no $v_{n}$-torsion. It is once again true that $J$ contains a nonzero subcomodule that is finitely generated over $B P_{*}$. The new ingredient is [Lan79, Corollary 7], which implies that, since $J$ has no $v_{n}$-torsion, its finitely generated subcomodules are in fact finitely presented over $B P_{*}$. Therefore we can use the usual Landweber filtration theorem [Lan76] to find a subcomodule of the form $\Sigma^{t} B P_{*} / I_{r}$ for $0 \leq r \leq n$. As before, this implies that $J \cong \Sigma^{t} J_{r}$.

Note that the obvious inclusions

$$
B P_{*} / I_{n} \rightarrow \Sigma^{t} B P_{*} /\left(p^{\infty}, \ldots, v_{n-1}^{\infty}\right) \rightarrow v_{n}^{-1} \Sigma^{t} B P_{*} /\left(p^{\infty}, \ldots, v_{n-1}^{\infty}\right)
$$

are essential extensions, so these important comodules (defined more precisely after Theorem 3.1) have the same injective hull $J_{n}$. Here $t=\left|v_{1}\right|+$ $\cdots+\left|v_{n-1}\right|$, and the inclusion sends $1 \in B P_{*} / I_{n}$ to $1 / p v_{1} \cdots v_{n-1}$.

It is also useful to know that $\Phi_{*}$ and $\Phi^{*}$ map these indecomposable injectives as one would expect.

Lemma 2.5. We have $\Phi_{*} J_{r} \cong J_{n, r}$ and $\Phi^{*} J_{n, r} \cong J_{r}$ for $0 \leq r \leq n$.

Proof. Recall that both $\Phi_{*}$ and $\Phi^{*}$ preserve injectives by [HS05b, Corollary 2.5], and $\Phi^{*}$ preserves indecomposables by Lemma 2.2. Hence $\Phi^{*} J_{n, r}$ is an indecomposable injective, necessarily without $v_{n}$-torsion. On the other hand, since

$$
\Phi_{*} B P_{*} / I_{r}=E_{*} / I_{r} \subseteq J_{n, r},
$$

we conclude that

$$
L_{n}\left(B P_{*} / I_{r}\right) \subseteq \Phi^{*} J_{n, r} .
$$

But $L_{n}\left(B P_{*} / I_{r}\right)$ is either $B P_{*} / I_{r}$ itself if $r<n$, or $v_{n}^{-1} B P_{*} / I_{n}$ if $r=n$ [HS05a, Lemma 5.3]. In either case we see that $J_{r} \subseteq \Phi^{*} J_{n, r}$. Equality must hold since $\Phi^{*} J_{n, r}$ is an indecomposable injective.

It then follows that $\Phi_{*} J_{r}=\Phi_{*} \Phi^{*} J_{n, r} \cong J_{n, r}$, as required.

This lemma implies that $J_{n, r}$ and $J_{r}$ share much of the same structure. For example, we have the following corollary.

Corollary 2.6. Suppose $0 \leq r \leq n$. Then $J_{r}$ and $J_{n, r}$ are $I_{r}$-torsion and $v_{r}$-periodic.

Proof. For $J_{r}$, this follows from Proposition 2.2, Proposition 2.6, and Theorem 2.7 of [HS05b]. It is clear that if $M$ is $I_{r}$-torsion, so is $\Phi_{*} M$, and so 
$J_{n, r}$ is $I_{r}$-torsion. In particular, since $v_{r}$ is a primitive modulo $I_{r}$, this means that $v_{r}^{-1} J_{n, r}$ is a well-defined comodule. The kernel $K$ of $J_{n, r} \rightarrow v_{r}^{-1} J_{n, r}$ is the $v_{r}$-torsion in $J_{r}$ and $K$ intersects $E_{*} / I_{r}$ trivially. Since $J_{n, r}$ is an essential extension of $E_{*} / I_{r}$, we conclude that $J_{n, r}$ has no $v_{r}$-torsion. This means that $v_{r}^{-1} J_{n, r}$ is an essential extension of $J_{n, r}$, so $v_{r}^{-1} J_{n, r}=J_{n, r}$.

3. Structure of indecomposable injectives. We would like to know more of the structure of the indecomposable injectives $J_{r}$ and $J_{n, r}$, in analogy to Matlis' theory described in [Lam99, Section 3I].

The object of this section is to prove the following theorem.

THEOREM 3.1. If $E=E(n)$, then there is an isomorphism of comodules

$$
J_{n, n} \cong E(n)_{*} E(n) \otimes_{E(n)_{*}} \Sigma^{t} E(n)_{*} / I_{n}^{\infty},
$$

where $t=\left|v_{1}\right|+\cdots+\left|v_{n-1}\right|$.

Note that in this theorem, we are not free to use any Noetherian Landweber exact homology theory of height $n$, but must use $E(n)$ itself. In particular, we cannot use Morava $E$-theory.

In this theorem, $E(n)_{*} / I_{n}^{\infty}$ is thought of as an $E(n)_{*}$-module, not as a comodule, and it is the usual construction used in algebraic topology. That is, we inductively define $E(n)_{*} / I_{r}^{\infty}$ via the short exact sequence

$$
0 \rightarrow E(n)_{*} / I_{r}^{\infty} \rightarrow v_{r}^{-1} E(n)_{*} / I_{r}^{\infty} \rightarrow E(n)_{*} / I_{r+1}^{\infty} \rightarrow 0 .
$$

Additive generators of $E(n)_{*} / I_{n}^{\infty}$ are given by

$$
\frac{\lambda v_{n}^{r}}{p^{i_{0}} v_{1}^{i_{1}} \cdots v_{n-1}^{i_{n-1}}}
$$

where $\lambda \in \mathbb{F}_{p}, r \in \mathbb{Z}$ and $i_{0}, i_{1}, \ldots, i_{n-1}$ are positive integers. The action of $p, v_{1}, \ldots, v_{n}$ is the obvious one, with a product being 0 if it ever removes any of $p, v_{1}, \ldots, v_{n-1}$ from the denominator. For example, $E(n)_{*} / I_{n}$ is the submodule of $\Sigma^{t} E(n)_{*} / I_{n}^{\infty}$ generated by $1 / p v_{1} \cdots v_{n-1}$.

We can make a similar construction to form $E_{n *} / I_{n}^{\infty}$, with

$$
E_{n *}=W \mathbb{F}_{q}\left[\left[u_{1}, \ldots, u_{n-1}\right]\right]\left[u, u^{-1}\right]
$$

the coefficient ring of Morava $E$-theory, where $q=p^{n}, W \mathbb{F}_{q}$ is the Witt vectors of the Galois field $\mathbb{F}_{q}$, the $u_{i}$ have degree 0 , and $u$ has degree -2 . There is a ring homomorphism $E(n)_{*} \rightarrow E_{n *}$ that takes $v_{r}$ to $u_{r} u^{1-p^{r}}$ for $1 \leq r<n$ and $v_{n}$ to $u^{1-p^{n}}$. Hence $I_{r}=\left(p, u_{1}, \ldots, u_{r-1}\right)$ as an ideal of $E_{n *}$. The elements

$$
\frac{\lambda u^{r}}{p^{i_{0}} u_{1}^{i_{1}} \cdots u_{n-1}^{i_{n-1}}},
$$

where $\lambda \in \mathbb{F}_{q}, r \in \mathbb{Z}$ and $i_{0}, i_{1}, \ldots, i_{n-1}$ are all positive integers, are additive generators for $E_{n *} / I_{n}^{\infty}$. 
It is important to note that $E(n)_{*} / I_{n}^{\infty}$ is not the increasing union of the $E(n)_{*} / I_{n}^{r}$ for $n>1$. Indeed, $E(n)_{*} / I_{n}^{2}$ has distinct elements $p$ and $v_{1}$ that are both killed by $I_{n}$, whereas the only elements of $E(n)_{*} / I_{n}^{\infty}$ killed by $I_{n}$ are $\alpha v_{n}^{r} / p v_{1} \cdots v_{n-1}$ for $\alpha \in \mathbb{F}_{p}$ and $r \in \mathbb{Z}$. Thus no shifted copy of $E(n)_{*} / I_{n}^{2}$ can sit inside $E(n)_{*} / I_{n}^{\infty}$ for $n>1$.

Theorem 3.1 has the following corollary.

Corollary 3.2. We have

$$
J_{r} \cong B P_{*} B P \otimes_{B P_{*}} \Sigma^{t} E(r)_{*} / I_{r}^{\infty}
$$

and, for any Noetherian Landweber exact $E$ of height $n$,

$$
J_{n, r} \cong E_{*} B P \otimes_{B P_{*}} \Sigma^{t} E(r)_{*} / I_{r}^{\infty}
$$

for $0 \leq r \leq n$. Here $t=\left|v_{1}\right|+\cdots+\left|v_{r-1}\right|$.

In this corollary, $E$ is, as always, a Noetherian Landweber exact homology theory of height $n$.

Proof. The corollary follows from the fact that

$$
\Phi^{*}\left(E_{*} E \otimes_{E_{*}} M\right)=B P_{*} B P \otimes_{B P_{*}} M
$$

(see Lemma 2.4 of [HS05a]), and Lemma 2.5.

We will prove Theorem 3.1 by first using Matlis theory to show that $\Sigma^{t} E(n)_{*} / I_{n}^{\infty}$ is the injective hull of $E(n)_{*} / I_{n}$ in the category of $E(n)_{*^{-}}$ modules. This shows that $E(n)_{*} E(n) \otimes_{E(n)_{*}} \Sigma^{t} E(n)_{*} / I_{n}^{\infty}$ is an injective comodule. We then show that both of the maps in the composition

$E(n)_{*} / I_{n} \stackrel{i}{\rightarrow} E(n)_{*} E(n) \otimes_{E(n)_{*}} E(n)_{*} / I_{n} \stackrel{1 \otimes j}{\longrightarrow} E(n)_{*} E(n) \otimes_{E(n)_{*}} \Sigma^{t} E(n)_{*} / I_{n}^{\infty}$ are essential extensions. Here $i$ is the unit of the adjunction between the forgetful functor and the extended comodule functor, with $i(a)=\eta_{L}(a) \otimes 1$, and $j$ is the embedding of the $E(n)_{*}$-module $E(n)_{*} / I_{n}$ into its injective hull.

Proposition 3.3. The injective hull of $E(n)_{*} / I_{n}$ in the category of $E(n)_{*}$-modules is $\Sigma^{t} E(n)_{*} / I_{n}^{\infty}$, where $t=\left|v_{1}\right|+\cdots+\left|v_{n-1}\right|$. Similarly, the injective hull of $E_{n *} / I_{n}$ in the category of $E_{n *}$-modules is $E_{n *} / I_{n}^{\infty}$.

Proof. The proof is a computation using Matlis theory, modeled on $\left[\right.$ Lam99, Section 3J]. We first note that $\mathbb{Q} / \mathbb{Z}_{(p)}\left[v_{n}, v_{n}^{-1}\right]$ is an injective object in the category of graded $\mathbb{Z}_{(p)}\left[v_{n}, v_{n}^{-1}\right]$-modules and graded homomorphisms (not necessarily of degree 0 ). To see this, one can just use the same proof as the proof that shows $\mathbb{Q} / \mathbb{Z}_{(p)}$ is an injective $\mathbb{Z}_{(p)}$-module. This proof works for discrete valuation rings such as $W \mathbb{F}_{q}$ as well, so that $\left(W \mathbb{F}_{q} \otimes \mathbb{Q}\right) / \mathbb{Q}\left[u, u^{-1}\right]$ is an injective object in the category of graded $W \mathbb{F}_{q}\left[u, u^{-1}\right]$-modules and graded homomorphisms. The rest of the proof for $E_{n *}$ is the same as the proof for $E(n)_{*}$ given below. 
Now let $M=\operatorname{Hom}_{\mathbb{Z}_{(p)}\left[v_{n}, v_{n}^{-1}\right]}^{*}\left(E(n)_{*}, \mathbb{Q} / \mathbb{Z}_{(p)}\left[v_{n}, v_{n}^{-1}\right]\right) ;$ then $M$ is an injective graded $E(n)_{*}$-module by the generalization of [Lam99, Lemma 3.5] to the graded case. We define the element

$$
\frac{v_{n}^{r}}{p^{i_{0}} v_{1}^{i_{1}} \cdots v_{n-1}^{i_{n-1}}}=\frac{1}{p^{i_{0}} v_{1}^{i_{1}} \cdots v_{n-1}^{i_{n-1}} v_{n}^{-r}}
$$

of $M$ to be the element that takes the monomial

$$
v_{1}^{i_{1}} \cdots v_{n-1}^{i_{n-1}}
$$

to $v_{n}^{r} / p^{i_{0}} \in \mathbb{Q} / \mathbb{Z}_{(p)}\left[v_{n}, v_{n}^{-1}\right]$, and takes the complementary $\mathbb{Z}_{(p)}\left[v_{n}, v_{n}^{-1}\right]$ summand of $E(n)_{*}$ to 0 . Then the submodule of $M$ generated by these elements is $E(n)_{*} / I_{n}^{\infty}$. An arbitrary element of $M$ can be written as an infinite sum of these elements.

By Proposition 3.88 of [Lam99] (again, in the graded case), the $I_{n}$-torsion in $M$ is still an injective $E(n)_{*}$-module. We claim that the $I_{n}$-torsion in $M$ is just $E(n)_{*} / I_{n}^{\infty}$. Indeed, it is clear that each of the elements

$$
\frac{v_{n}^{r}}{p^{i_{0}} v_{1}^{i_{1}} \cdots v_{n-1}^{i_{n-1}}}
$$

is $I_{n}$-torsion. On the other hand, if $f \in M$ is $I_{n}$-torsion, then $I_{n}^{k} f=0$ for large $k$, so $f\left(I_{n}^{k}\right)=0$. Thus $f$ kills all but finitely many monomials $p^{i_{0}} v_{1}^{i_{1}} \cdots v_{n-1}^{i_{n-1}}$ in $E(n)_{*}$. It follows that $f$ is a finite sum of our generating elements, so is in $E(n)_{*} / I_{n}^{\infty}$.

Now it is clear that the extension $\Sigma^{-t} E(n)_{*} / I_{n} \rightarrow E(n)_{*} / I_{n}^{\infty}$, where 1 goes to $1 / p v_{1} \cdots v_{n-1}$, is essential, completing the proof.

We need a simple test for essential extensions of comodules.

Lemma 3.4. Suppose $(A, \Gamma)$ is a flat Hopf algebroid for which every $\Gamma$-comodule has a primitive. Then an extension $M \rightarrow N$ of $\Gamma$-comodules is essential if and only if $A x \cap M \neq 0$ for all primitives $x \in N$.

Note that every $E_{*} E$-comodule has a primitive by [HS05a, Theorem 5.1].

Proof. If $N^{\prime}$ is an arbitrary nonzero subcomodule of $N$, it must contain a primitive $x$ of $N$. The subcomodule $A x$ generated by $x$ is then inside $N^{\prime}$. The result follows easily.

Lemma 3.5. If $(A, \Gamma)$ is a flat Hopf algebroid and $M$ is an $A$-module, then the primitives in the extended comodule $\Gamma \otimes_{A} M$ are the elements $1 \otimes m$.

Proof. Note that $x$ is a primitive if and only if $x=f(1)$ for some comodule map $f: A \rightarrow \Gamma \otimes_{A} M$. But then adjointness implies $f$ is induced by a map $g: A \rightarrow M$ of $A$-modules. This means that $f(a)=\eta_{L}(a) \otimes g(a)$ for all $a \in A$. In particular, $x=f(1)=1 \otimes g(1)$. The converse is clear. 
Proposition 3.6. The map

$$
E(n)_{*} / I_{n} \stackrel{i}{\rightarrow} E(n)_{*} E(n) \otimes_{E(n)_{*}} E(n)_{*} / I_{n}
$$

defined by $i(a)=\eta_{L}(a) \otimes 1$ is an essential extension of $E(n)_{*} E(n)$-comodules.

We note that this proposition is also true for Morava $E$-theory $E_{n}$, but the proof requires more care.

Proof. The primitives in $E(n)_{*} E(n) \otimes_{E(n)_{*}} E(n)_{*} / I_{n}$ are the elements $1 \otimes \bar{\lambda} v_{n}^{k}$ for $\lambda \in \mathbb{Z}_{(p)}$ (so $\bar{\lambda}$ is the reduction of $\lambda$ in $\mathbb{F}_{p}$ ) and $k \in \mathbb{Z}$ by Lemma 3.5. But we have

$$
1 \otimes \bar{\lambda} v_{n}^{k}=\eta_{R}(\lambda) \eta_{R}\left(v_{n}\right)^{k} \otimes 1 .
$$

Of course $\eta_{R}(\lambda)=\lambda$, and also $\eta_{R}\left(v_{n}\right) \equiv v_{n}\left(\bmod I_{n}\right)$. Hence

$$
1 \otimes \bar{\lambda} v_{n}^{k}=\eta_{L}\left(\lambda v_{n}^{k}\right) \otimes 1 .
$$

Lemma 3.4 now completes the proof.

Proposition 3.7. The map

$$
E(n)_{*} E(n) \otimes_{E(n)_{*}} E(n)_{*} / I_{n} \stackrel{1 \otimes j}{\longrightarrow} E(n)_{*} E(n) \otimes_{E(n)_{*}} \Sigma^{t} E(n)_{*} / I_{n}^{\infty}
$$

is an essential extension of $E(n)_{*} E(n)$-comodules. Here $t=\left|v_{1}\right|+\cdots+\left|v_{n-1}\right|$.

Note that this proposition completes the proof of Theorem 3.1. It is this proposition that we believe to be false for Morava $E$-theory $E_{n}$.

Proof. In view of Lemmas 3.4 and 3.5, it suffices to show that there is an $a \in E(n)_{*}$ such that

$$
\eta_{L}(a) \otimes \frac{\lambda v_{n}^{k}}{p^{i_{0}} v_{1}^{i_{1}} \cdots v_{n-1}^{i_{n-1}}}=1 \otimes \frac{\lambda v_{n}^{k}}{p v_{1} \cdots v_{n-1}},
$$

ignoring suspensions. But then it is clear that we should take

$$
a=p^{i_{0}-1} v_{1}^{i_{1}-1} \cdots v_{n-1}^{i_{n-1}-1} .
$$

There is a slight subtlety, since $\eta_{L}\left(v_{j}\right) \neq \eta_{R}\left(v_{j}\right)$. We get around this by going in order, from $p$ to $v_{1}$ to $v_{2}$, et cetera, using the fact that $v_{j}$ is primitive modulo $I_{j}$. More precisely, for the inductive step, we have

$$
\begin{aligned}
x \eta_{L}\left(v_{j}^{i_{j}-1}\right) \otimes \frac{v_{n}^{k}}{p v_{1} \cdots v_{j-1} v_{j}^{i_{j}} \cdots v_{n-1}^{i_{n-1}}} & =x \eta_{R}\left(v_{j}^{i_{j-1}}\right) \otimes \frac{v_{n}^{k}}{p v_{1} \cdots v_{j-1} v_{j}^{i_{j}} \cdots v_{n-1}^{i_{n-1}}} \\
& =x \otimes \frac{v_{n}^{k}}{p v_{1} \cdots v_{j} v_{j+1}^{i_{j+1}} \cdots v_{n-1}^{i_{n-1}}},
\end{aligned}
$$

as required. 
4. Indecomposable injectives and duality. In this section, we study the dualities in the stable homotopy category that arise from the indecomposable injectives studied in this paper.

Fix a $p$-local spectrum $X$, and integers $n \geq r$, and consider the functor that takes a $p$-local spectrum $Y$ to the abelian group $\operatorname{Hom}_{E_{*} E}\left(E_{*}(X \wedge Y), J_{n, r}\right)$, where $E$ is a Landweber exact homology theory of height $n$, as usual. Since $J_{n, r}$ is an injective $E_{*} E$-comodule, this functor is exact and so is a cohomology theory of $Y$. Hence it is representable by some spectrum $\Delta_{n, r} X$, for which we have the natural isomorphism

$$
\left[Y, \Delta_{n, r} X\right] \cong \operatorname{Hom}_{E_{*} E}\left(E_{*}(X \wedge Y), J_{n, r}\right) .
$$

Using the injective $B P_{*} B P$-comodule $J_{r}$, we can also define $\Delta_{r} X$ with the property that

$$
\left[Y, \Delta_{r} X\right] \cong \operatorname{Hom}_{B P_{*} B P}\left(B P_{*}(X \wedge Y), J_{r}\right) .
$$

We have the following basic results about these duality functors.

Proposition 4.1. For a p-local spectrum $X$, define $\Delta_{n, r} X$ and $\Delta_{r} X$ as above. Denote $\Delta_{n, r} S^{0}$ and $\Delta_{r} S^{0}$ by $\Delta_{n, r}$ and $\Delta_{r}$. Then:

(1) $\Delta_{n, r} X \cong \Delta_{r} X$ for all $n \geq r$, and so $\Delta_{n, r}$ is independent of the choice of $E$.

(2) $\left[Y, \Delta_{r} X\right] \cong \operatorname{Hom}_{E(r)_{*}}\left(E(r)_{*}(X \wedge Y), \Sigma^{t} E(r)_{*} / I_{r}^{\infty}\right)$, where $t=\left|v_{1}\right|+$ $\cdots+\left|v_{n-1}\right|$.

(3) $\Delta_{r} X \cong F\left(X, \Delta_{r}\right)$.

(4) $\Delta_{r}$ is a BP-injective spectrum with $B P_{*} \Delta_{r} \cong J_{r}$, and the isomorphism

$$
\left[X, \Delta_{r}\right] \cong \operatorname{Hom}_{B P_{*} B P}\left(B P_{*} X, J_{r}\right)
$$

is induced by taking BP-homology. If $E_{*}$ is concentrated in even dimensions, then $\Delta_{r}$ is also an E-injective spectrum for all $n \geq r$, with $E_{*} \Delta_{r} \cong J_{n, r}$, and the isomorphism

$$
\left[X, \Delta_{r}\right] \cong \operatorname{Hom}_{E_{*} E}\left(E_{*} X, J_{n, r}\right)
$$

is induced by taking E-homology.

(5) $\Delta_{r} X$ is $L_{r}$-local. In particular, $\Delta_{r} X=F\left(L_{r} X, \Delta_{r}\right)$.

(6) $\Delta_{r} X=0$ if and only if $L_{r} X=0$.

Proof. For part (1), we have

$$
\begin{aligned}
{\left[Y, \Delta_{n, r} X\right] } & =\operatorname{Hom}_{E(n)_{*} E(n)}\left(E(n)_{*}(X \wedge Y), J_{n, r}\right) \\
& \cong \operatorname{Hom}_{B P_{*} B P}\left(B P_{*}(X \wedge Y), \Phi^{*} J_{n, r}\right) \\
& \cong \operatorname{Hom}_{B P_{*} B P}\left(B P_{*}(X \wedge Y), J_{r}\right)=\left[Y, \Delta_{r} X\right],
\end{aligned}
$$

using the adjointness between $\Phi_{*}$ and $\Phi^{*}$ and the isomorphism $\Phi^{*} J_{n, r}=J_{r}$ of Lemma 2.5. 
Part (2) follows from part (1) and the isomorphisms

$$
\begin{aligned}
{\left[Y, \Delta_{r, r} X\right] } & =\operatorname{Hom}_{E(r)_{*} E(r)}\left(E(r)_{*}(X \wedge Y), J_{r, r}\right) \\
& \cong \operatorname{Hom}_{E(r)_{*}}\left(E(r)_{*}(X \wedge Y), E(r)_{*} / I_{r}^{\infty}\right),
\end{aligned}
$$

the last of which follows from Theorem 3.1.

Part (3) is an exercise in adjointness, and part (4) follows from [Dev97, Proposition 1.3 and Theorem 1.5].

Part (5) is clear, since if $Y$ is $L_{r}$-acyclic, then $E(r)_{*}(X \wedge Y)=0$ and so $\left[Y, \Delta_{r} X\right]=0$.

For part (6), suppose $L_{r} X \neq 0$. Then $E(r)_{*} X \neq 0$, and so $E(r)_{*} X$ contains a subcomodule $M$ isomorphic to $\Sigma^{r} E(r)_{*} / I_{j}$ for some $0 \leq j \leq r$ and some $r$ by [HS05a, Theorem 5.7]. There is obviously a nonzero map (of some degree) $M \rightarrow E(r)_{*} / I_{r}^{\infty}$ of $E(r)_{*}$-modules, obtained by modding out by $I_{r}$ and including. Since $E(r)_{*} / I_{r}^{\infty}$ is injective by Proposition 3.3, this map extends to a nonzero map (of some degree) $E(r)_{*} X \rightarrow E(r)_{*} / I_{r}^{\infty}$. This map corresponds to a nonzero homotopy class in $\Delta_{r} X$, and so $\Delta_{r} X$ is nonzero.

We also point out that, as usual, there is a natural map $X \rightarrow \Delta_{r}^{2} X$ obtained by taking the image of the identity under the isomorphisms

$$
\left[\Delta_{r} X, \Delta_{r} X\right] \cong \operatorname{Hom}_{E(r)_{*}}\left(E(r)_{*}\left(X \wedge \Delta_{r} X\right), \Sigma^{t} E(r)_{*} / I_{r}^{\infty}\right) \cong\left[X, \Delta_{r}^{2} X\right] .
$$

The spectrum $\Delta_{r}$ has in fact been studied before in stable homotopy theory. Recall that $L_{n}$ denotes Bousfield localization with respect to $E(n)$, or any Landweber exact theory of height $n$. There is a natural map $L_{n} X \rightarrow$ $L_{n-1} X$, and the homotopy fiber of this map is traditionally denoted $M_{n} X$.

Theorem 4.2. Fix an integer $r \geq 1$. Then $\Delta_{r} \cong \Sigma^{t+r} M_{r} E(r)$, where $t=\left|v_{1}\right|+\cdots+\left|v_{r-1}\right|$. In particular, if $E_{*}$ is an evenly graded Landweber exact homology theory of height $n \geq r$, then

$$
J_{n, r} \cong \Sigma^{t+r} E_{*}\left(M_{r} E(r)\right) .
$$

Proof. Recall from [Rav84, Theorem 6.1] that

$$
\pi_{*} M_{r} B P \cong \Sigma^{-r} v_{r}^{-1}\left(B P_{*} / I_{r}^{\infty}\right) .
$$

In view of the localization theorem [Rav92, Theorem 7.5.2], we see that

$$
B P_{*} M_{r} S^{0} \cong \Sigma^{-r} v_{r}^{-1}\left(B P_{*} / I_{r}^{\infty}\right) .
$$

By Landweber exactness, we conclude that

$$
E(r)_{*} M_{r} S^{0} \cong \Sigma^{-r} E(r)_{*} / I_{r}^{\infty} .
$$

But $M_{r} E(r) \cong E(r) \wedge M_{r} S^{0}$ because of the smash product theorem [Rav92, Theorem 7.5.6], and the flatness of $E(r)_{*} E(r)$ implies that

$$
E(r)_{*}(E(r) \wedge X) \cong E(r)_{*} E(r) \otimes_{E(r)_{*}} E(r)_{*} X
$$


(see Lemma 2.2.7 of [Rav86]). Some diagram chasing is necessary to show that this isomorphism is an isomorphism of comodules, where the right side is given the extended comodule structure, so that all the coaction is on the $E(r)_{*} E(r)$ tensor factor. Theorem 3.1 then implies that

$$
E(r)_{*}\left(M_{r} E(r)\right) \cong \Sigma^{-t-r} J_{r, r}
$$

as $E(r)_{*} E(r)$-comodules. This isomorphism then defines a map

$$
M_{r} E(r) \rightarrow \Sigma^{-t-r} \Delta_{r}
$$

that is an isomorphism in $E(r)$-homology. Since both $M_{r} E(r)$ and $\Delta_{r}$ are $E(r)$-local, the theorem follows.

This theorem has the following corollary, apparently not written down before but known to several mathematicians, including Hopkins, Greenlees, and Sadofsky.

Corollary 4.3. Let $\widehat{E(n)}$ denote $L_{K(n)} E(n)$, the completion of $E(n)$ at $I_{n}$. Then

$$
\widehat{E(n)}^{*}(X) \cong \operatorname{Hom}_{E(n)_{*}}\left(E(n)_{*}\left(M_{n} X\right), \Sigma^{-n} E(n)_{*} / I_{n}^{\infty}\right) .
$$

On the simplest level, we can think of this corollary as analogous to the fact that $\operatorname{Hom}_{\mathbb{Z}_{(p)}}\left(\mathbb{Q} / \mathbb{Z}_{(p)}, \mathbb{Q} / \mathbb{Z}_{(p)}\right) \cong \mathbb{Z}_{(p)}$. As pointed out by Greenlees, one can look at this corollary as a reflection of the fact that completion (represented by $\widehat{E(n)}$ ) should be maps out of local cohomology (represented by $\left.M_{n} E(n)\right)$. This is the viewpoint of [GM95, Section 4].

Proof. Theorem 6.19 of [HS99] tells us that

$$
\widehat{E(n)}^{*}(X) \cong\left[L_{K(n)} X, L_{K(n)} E(n)\right]^{*} \cong\left[M_{n} X, M_{n} E(n)\right]^{*} .
$$

But Theorem 4.2 implies that

$$
\begin{aligned}
{\left[M_{n} X, M_{n} E(n)\right]^{*} } & \cong\left[\Sigma^{t+n} M_{n} X, \Delta_{n}\right]^{*} \\
& \cong \operatorname{Hom}_{E(n)_{*}}\left(E(n)_{*}\left(\Sigma^{t+n} M_{n} X\right), \Sigma^{t} E(n)_{*} / I_{n}^{\infty}\right) \\
& \cong \operatorname{Hom}_{E(n)_{*}}\left(E(n)_{*} M_{n} X, \Sigma^{-n} E(n)_{*} / I_{n}^{\infty}\right) .
\end{aligned}
$$

We can also determine the endomorphism rings of the indecomposable injectives $J_{r}$ and $J_{n, r}$.

COROLlary 4.4. The endomorphism rings $\operatorname{End}_{B P_{*} B P}\left(J_{r}\right)$ and $\operatorname{End}_{E(n)_{*} E(n)}\left(J_{n, r}\right)$ for $0 \leq r \leq n$ are isomorphic to $\widehat{E(r)}^{*} \widehat{E(r)}$.

Proof. Both of these endomorphism rings are isomorphic to $\left[\Delta_{r}, \Delta_{r}\right]^{*}$ in view of Proposition 4.1(4). But Theorem 4.2 implies that

$$
\left[\Delta_{r}, \Delta_{r}\right]^{*} \cong\left[M_{r} E(r), M_{r} E(r)\right]^{*},
$$

which is in turn isomorphic to $\widehat{E(r)} * \widehat{E(r)}$ by [HS99, Theorem 6.19]. 
It is unfortunate that these endomorphism rings of indecomposable injectives turn out to be $\widehat{E(r)}^{*} \widehat{E(r)}$ instead of $E_{r}^{*} E_{r}$, where $E_{r}$ is Morava $E$-theory. The ring $E_{r}^{*} E_{r}$ of operations in Morava $E$-theory is the twisted completed group ring $E_{r *}[[\Gamma]]$, where $\Gamma$ is the semidirect product of the automorphism group of the height $r$ Honda formal group law over $\mathbb{F}_{p^{r}}$ with the Galois group of $\mathbb{F}_{p^{r}}$. This is an old result of Hopkins and Ravenel; see [Bak95] or [Hov04b] for a proof. Since $E_{r}$ is a finite free module over $\widehat{E(r)}$, there is probably some Galois theory relating $\widehat{E(r)}^{*} \widehat{E(r)}$ to $E_{r *}[[\Gamma]]$, but the author does not know any details.

We can, however, make a decomposable injective comodule whose endomorphism ring is $E_{r}^{*} E_{r} \cong E_{r *}[[\Gamma]]$. Indeed, we can simply take the comodule

$$
J_{r, r}^{\prime}=E_{r *} E_{r} \otimes_{E_{r *}} E_{r *} / I_{r}^{\infty} .
$$

This is an $E_{r *} E_{r}$-comodule that is injective because $E_{r *} / I_{r}^{\infty}$ is an injective $E_{r *}$-module by Proposition 3.3. Under the equivalence of categories between $E(r)_{*} E(r)$-comodules and $E_{r *} E_{r}$-comodules of [HS05a], $J_{r, r}^{\prime}$ corresponds to the direct sum of $r\left(p^{r}-1\right)$ copies of $J_{r, r}$, with $r$ copies in every even dimension from 2 to $2\left(p^{r}-1\right)$; this is the $E(r)_{*} E(r)$-comodule

$$
E(r)_{*} E(r) \otimes_{E(r)_{*}} E_{r *} / I_{r}^{\infty} .
$$

There is a spectrum $\Delta_{r}^{\prime}$ with

$$
\left[X, \Delta_{r}^{\prime}\right] \cong \operatorname{Hom}_{E_{r *} E_{r}}\left(E_{r *} X, J_{r, r}^{\prime}\right) \cong \operatorname{Hom}_{E_{r *}}\left(E_{r *} X, E_{r *} / I_{r}^{\infty}\right) .
$$

The analogue to Theorem 4.2 tells us that $\Delta_{r}^{\prime} \cong \Sigma^{r} M_{r} E_{r}$. We then get isomorphisms

$$
E_{n}^{*} X \cong \operatorname{Hom}_{E_{n *}}\left(E_{n *}\left(M_{n} X\right), \Sigma^{-n} E_{n *} / I_{n}^{\infty}\right),
$$

again known before by Hopkins, Greenlees, Sadofsky, and others, and

$$
\operatorname{End}_{E_{r *} E_{r}}\left(J_{r, r}^{\prime}\right) \cong E_{r}^{*} E_{r} \cong E_{r *}[[\Gamma]]
$$

\section{References}

[Bak89] A. Baker, Some families of operations in Morava K-theory, Amer. J. Math. 111 (1989), 95-109.

[Bak95] - A version of Landweber's filtration theorem for $v_{n}$-periodic Hopf algebroids, Osaka J. Math. 32 (1995), 689-699.

[Dev97] E. S. Devinatz, Morava modules and Brown-Comenetz duality, Amer. J. Math. 119 (1997), 741-770.

[DH95] E. S. Devinatz and M. J. Hopkins, The action of the Morava stabilizer group on the Lubin-Tate moduli space of lifts, ibid. 117 (1995), 669-710.

[GM95] J. P. C. Greenlees and J. P. May, Completions in algebra and topology, in: Handbook of Algebraic Topology, North-Holland, Amsterdam, 1995, 255-276. 
[Hov95] M. Hovey, Bousfield localization functors and Hopkins' chromatic splitting conjecture, in: The Čech Centennial (Boston, MA, 1993), Contemp. Math. 181, Amer. Math. Soc., Providence, RI, 1995, 225-250.

[Hov04a] - Homotopy theory of comodules over a Hopf algebroid, in: Homotopy Theory: Relations with Algebraic Geometry, Group Cohomology, and Algebraic $K$-theory (Evanston, IL, 2002), Contemp. Math. 346, Amer. Math. Soc., Providence, RI, 2004, 261-304.

[Hov04b] - Operations and co-operations in Morava E-theory, Homology Homotopy Appl. 6 (2004), 201-236.

[Hov07] - The generalized homology of products, Glasg. Math. J. 49 (2007), 1-10.

[HS99] M. Hovey and N. P. Strickland, Morava K-theories and localisation, Mem. Amer. Math. Soc. 139 (1999), no. 666.

[HS05a] - - Comodules and Landweber exact homology theories, Adv. Math. 192 (2005), 427-456.

[HS05b] - - - Local cohomology of BP*BP-comodules, Proc. London Math. Soc. 90 (2005), 521-544.

[Lam99] T. Y. Lam, Lectures on Modules and Rings, Springer, New York, 1999.

[Lan73] P. S. Landweber, Annihilator ideals and primitive elements in complex bordism, Illinois J. Math. 17 (1973), 273-284.

[Lan76] - Homological properties of comodules over $M U_{*}(M U)$ and $B P_{*}(B P)$, Amer. J. Math. 98 (1976), 591-610.

[Lan79] - New applications of commutative algebra to Brown-Peterson homology, in: Algebraic Topology (Waterloo, ON, 1978), Lecture Notes in Math. 741, Springer, Berlin, 1979, 449-460.

[Rav84] D. C. Ravenel, Localization with respect to certain periodic homology theories, Amer. J. Math. 106 (1984), 351-414.

[Rav86] - Complex Cobordism and Stable Homotopy Groups of Spheres, Pure Appl. Math. 121, Academic Press, Orlando, FL, 1986.

[Rav92] - Nilpotence and periodicity in stable homotopy theory, Ann. of Math. Stud. 128, Princeton Univ. Press, Princeton, NJ, 1992.

[Ste75] B. Stenström, Rings of Quotients. An Introduction to Methods of Ring Theory, Grundlehren Math. Wiss. 217, Springer, Berlin, 1975.

Department of Mathematics

Wesleyan University

Middletown, CT 06459, U.S.A.

E-mail: hovey@member.ams.org

Received 27 March 2006;

in revised form 23 May 2007 